\title{
Infrared light as a potential therapeutic approach for neurodegeneration
}

\author{
Kazuhiro Nakamura ${ }^{1 *}$ and Takayasu Kawasaki ${ }^{2 *}$ \\ ${ }^{1}$ Department of Laboratory Sciences, Gunma University Graduate School of Health Sciences, Japan \\ ${ }^{2}$ IR Free Electron Laser Research Center, Research Institute for Science and Technology, Organization for Research Advancement, Tokyo University of Science, \\ Japan
}

\begin{abstract}
Neurodegenerative disorders such as Alzheimer's disease and Huntington's disease are devastating diseases that lead to cognitive and/or behavioral impairments. The misfolded causative molecules of these disorders form toxic aggregates that eventually induce synaptic dysfunction and neuronal cell death. To date, several drugs have been demonstrated to be beneficial for a substantial number of patients by improving their behavioral signs and symptoms and cognition. However, these drugs essentially target symptomatic treatments and leave the toxic aggregates intact. As the result, the effects of these drugs last only for a short period of time after drug administration. Recently, laser irradiations with near- and mid-infrared light have been tested to cultured cells and model animals of neurodegenerative disorders. The beneficial effects of the laser irradiation include restoration of intracellular organelle and dissociation of toxic aggregates. Laser irradiation might have a potential to be applied for treatments of multiple neurodegenerative disorders provided that appropriate dosages and their safety are thoroughly established.
\end{abstract}

\section{Abbreviations}

A $\beta$ :Amyloid beta; AD: Alzheimer's disease; APP:Amyloid precursor protein; BDNF:Brain-derived neurotrophic factor; CNS:Central nervous system; FEL:Free electron laser; MSCs:Mesenchymal stem cells; PS1:Presenilin 1; SCA:Spinocerebellar ataxia; ThS:Thioflavin-S.

\section{Alzheimer's disease \\ Deposit of aggregated amyloid beta $(A \beta)$ and hyperphosphorylated tau are major cause of Alzheimer's disease (AD). Therefore, peptides to interfere aggregation of $A \beta$ might theoretically restore toxic effects of $A \beta[1-3]$. In addition, laser irradiation to the brain to dissociate the aggregate is an attractive procedure because that is not interrupted by blood brain barrier.}

Low-level laser irradiation applies near-infrared light (600-1000 $\mathrm{nm}$ ) [4] to deliver low energy, non-heating infrared light into tissues deeply and is suggested to reduce inflammation and edema and also to promote healing of wounds, deeper tissues and nerves[5]. Therefore, low-level laser irradiation might be potentially applied to stroke, heart attack, spinal cord injury, and traumatic brain injury (reviewed in [5]). As the mechanisms by which low-level laser irradiation exerts beneficial effects on diseases, low-level laser irradiation restores functions of mitochondria, modulates reactive oxygen species and induces transcription factors (reviewed in [5]).

Meng et al. found that low-level laser therapy rescued neurons loss and dendritic atrophy in both $A \beta$-treated hippocampal neurons and cultured hippocampal neurons from amyloid precursor protein (APP)/ presenilin 1 (PS1) AD model mouse. As a mechanism, low-level laser therapy upregulated brain-derived neurotrophic factor (BDNF) by activation of ERK/CREB pathway [6].

Near-infrared light was also transcranially applied to APP/ PS1 transgenic mouse brain as well. A $\beta$ plaque were reduced in the neocortex, hippocampus and cerebellum of near- infrared light-treated APP/PS1 mice compared to that of sham-treated mice[4,7]. In these studies, the authors also examined the effect of near-infrared light on tau pathology in K369I tau transgenic mice. Near-infrared light attenuated formation of neurofibrillary tangles and the hyperphosphorylation of tau in these brain regions.

Lu et al. [8]applied low-level laser irradiation in a different animal. They used a rat model of $\mathrm{AD}$ in which $\mathrm{A} \beta$ 1-42 was injected into bilateral hippocampal CA1 region. They irradiated a diode laser with continuous wave at $808 \mathrm{~nm}$ transcranially by focusing the beam from the fiber optic on the top of the rat head with shaved scalp. Treatment with low-level laser irradiation restored multiple mitochondrial functions. That altered fission and fusion protein levels and restored mitochondrial dynamics. Low-level laser irradiation also changed mitochondrial membrane potential, oxidized mitochondrial DNA, excessive mitophagy and mitochondrial homeostasis. As the result, low-level laser irradiation enhanced the total antioxidant capacity of neurons in the hippocampal CA1 subregion, while that reduced the oxidative damage. Likewise, that reduced $\mathrm{A} \beta$-induced reactive gliosis and inflammation as well as tau hyperphosphorylation as evidenced

Correspondence to: Kazuhiro Nakamura, Department of Laboratory Sciences, Gunma University Graduate School of Health Sciences, 3-39-22, showa-machi, Maebashi, Gunma 371-8511, Japan, E-mail: knakamur@gunma-u.ac.jp

Takayasu Kawasaki, IR Free Electron Laser Research Center, Research Institute for Science and Technology, Organization for Research Advancement, Tokyo University of Science, 2641, Yamazaki, Noda, Chiba 278-8510, Japan. E-mail: kawasaki@rs.tus.ac.jp

Key words: neurodegeneration, infrared

Received: February 10, 2017; Accepted: February 23, 2017; Published: February 28, 2017 
by PHF1 expression. Thus, low-level laser irradiation attenuated A $\beta$ induced hippocampal neurodegeneration and furthermore, restored long-term spatial and recognition memory impairments, as proved by Barnes maze task and novel object recognition test[8].

Low-level laser was also irradiated to bone marrow of 5xFAD transgenic AD model mouse line that overexpresses both familial AD mutant forms of APP (K670N/M671L, I716V and V717I) and PS1 (M146L/L286V) transgenes. The treatment significantly decreased A $\beta$ area burden in the hippocampus and improved cognitive capacity and spatial learning of the AD mice compared to non-treated AD mice [9].

In contrast to non-heating irradiation above, photothermal treatment using graphene oxide (GO) and near-infrared laser was employed to dissociate $A \beta$ fibrils [10]. This strategy utilizes nearinfrared optical absorption ability of nano-GO to generate local heat to dissociate $A \beta$ fibrils following low-power near-infrared laser irradiation $(808 \mathrm{~nm})$. To specifically target $\mathrm{A} \beta$ aggregates, $\mathrm{GO}$ was covalently linked to thioflavin-S (ThS), which can selectively bind to A $\beta$ aggregates. Upon near-infrared irradiation, the GO-ThS complex successfully dissociated amyloid deposits both in buffer and in mouse cerebrospinal fluid. Regarding cellular toxicity of $A \beta, A \beta$ fibrils led to a decrease of survival of PC12 cells using MTT assay. After treatment with GO-ThS followed by near-infrared laser irradiation, survival of the cells increased [10].

Based on the positive results of near-infrared light therapy for $\mathrm{AD}$ model animals, the first completed report of near-infrared photobiomodulation therapy for 5 cases with mild to moderately severe dementia and possible $\mathrm{AD}$ were published recently.The devices used are $810 \mathrm{~nm}, 10 \mathrm{~Hz}$ pulsed, light-emitting diode devices combining transcranial plus intranasal photobiomodulation. Increased function, better sleep, fewer angry outbursts, less anxiety, and wandering were reported. There were no negative side effects[11].Larger studies will determine its clinical potential.

\section{Polyglutamine disease}

A free electron laser (FEL) consists of an electron beam propagating through a periodic magnetic field and are capable of operation over the entire electromagnetic spectrum (reviewed in [12]).The mid-infrared FEL facility at the Tokyo University of Science can generate a laser beam using synchrotron radiation as a seed, with a variable wavelength within the mid-infrared region of 5-16 $\mu \mathrm{m}\left(625-2,000 \mathrm{~cm}^{-1}\right)$ [13]. We introduce dissociation of polyglutamine aggregate as an example demonstrating potential usefulness of mid-infrared wavelength by FEL below.

Spinocerebellar ataxia (SCA) is a major neurodegenerative disorder among over 50 different types of inherited ataxias [14,15]. There are various types of the SCA such as autosomal-dominantly inherited polyglutamine diseases in which a CAG trinucleotide repeat is expanded in the coding region of causative genes. For example, ataxin-1 protein (ATXN1) with an abnormally expanded polyglutamine stretch [16] leads to SCA type 1 (SCA1) that is characterized by neurodegeneration in broad regions in the central nervous system (CNS) including the cerebral cortex, basal ganglia, brainstem and cerebellum as well as in the spinal cord [17]. The pathogenesis of SCA1 is neuronal degeneration induced by aggregates of ATXN1 with an abnormally expanded polyglutamine stretch.

Although no fundamental treatment has been established for blocking or even delaying the progression of SCA symptoms, a biochemical approach using mesenchymal stem cells (MSCs) is currently being tested in clinical trials for SCA as well as for other nervous system diseases [18] such as multiple sclerosis [19] and spinal cord injury [20]. In a study using 14 cases of SCA patients [21], the intrathecal injection of umbilical cord MSCs could improve the movement ability and quality of daily life 1 month after the treatment, as evidenced by scores of the International Cooperative Ataxia Rating Scale (ICARS) and Activity of Daily Living Scale (ADL). In another study using intravenous and intrathecal infusion of umbilical cord MSCs [22], the majority of patients including SCA1, SCA2 and SCA3 showed improved scores on the Berg Balance Scale (BBS) and ICARS up to at least 6 months after the treatment. Also, the infusion proved safe based on the laboratory examinations[22]. Therefore, it seems that umbilical cord MSCs moderately improve the movement ability and quality of daily life of SCA patients in the absence of serious side effects.

Past studies using MSCs and SCA model mice provide us with good information on the mechanism how MSCs give beneficial effects to SCA patients. As an effect of MSCs to CNS in SCA animals, intrathecal injection of only $3 \times 10^{3}$ MSCs greatly mitigated the cerebellar neuronal disorganization such as multi-layer arrangement and atrophy of dendrites of Purkinje cells observed in SCA1-transgenic mice $[23,24]$. Furthermore, MSCs normalized deficits in motor coordination in the SCA1- transgenic mice $[23,24]$. MSCs even proved effective for attenuation of degeneration of spinal cord of SCA1-knockin (SCA1$\mathrm{KI}$ ) mice because MSCs led to a significant suppression of degeneration of both motor neuron axons and myelin sheaths, even 6 months following the MSC injection into SCA1-KI mice [25]. Notably, the therapeutic effects of MSCs in the SCA1-KI mice were reproduced with MSC-conditioned media (MSC-CM). MSC-CM morphologically attenuated the degeneration of axons and myelin of spinal neurons, and functionally, the injected SCA1-KI mice showed smaller reductions in nerve conduction velocity in spinal motor neurons and reduced motor incoordination than the untreated mice [26]. These findings indicate that the mechanism that underlies improvement of the movement ability of SCA patients is not dissociation of ATXN1 aggregates but likely to be trophic effect for neurons by factor(s) released from MSCs.

The aggregates in neurons interfere with normal neuronal functions. When we consider therapeutic approaches aiming at direct dissociation of toxic aggregates in neurons, laser with optimal wavelengths would be an attractive tool. The initial idea was brought by an observation that multiple protein aggregates can absorb most photon energy at the amide I band. Indeed, the structures of aggregated proteins was changed by a FEL tuned to the amide bands [13,27-30].

In the brain, aggregates consisting of amyloid $\beta$ and tau in Alzheimer's disease and those of polyglutamine-containing proteins exhibit $\beta$-sheet structures. In organs other than the brain, insulin as well as lysozyme, calcitonin, lactoferrin, $\beta_{2}$-microglobulin, and immunoglobulin light and heavy chains can form aggregates as well. Tiny strings of insulin fibrils prepared in an acidic solution (20\% acetic acid) decreased substantially after irradiation at $6.17 \mu \mathrm{m}\left(1620 \mathrm{~cm}^{-1}\right)$, which corresponds to the amide I band. After 1 hour of irradiation, the $\beta$-sheet content was decreased from $40 \%$ to $25 \%$, indicating that $\beta$-sheet rich structure was reduced by FEL irradiation at the amide I band [13]. Almost same wavelength was also effective to dissociate non-amyloid keratin aggregate [30]. The keratin aggregate before irradiation was rich in $\alpha$-helix [30]. Secondary structure analysis suggested that the $\alpha$-helix content in the aggregate structure reduced to a level almost same as the level in the monomer state after irradiation at $6.06 \mu \mathrm{m}$. Both irradiations at $6.51 \mu \mathrm{m}$ (amide II band) and $8.06 \mu \mathrm{m}$ (amide III band) were effective to a lesser extent than irradiation at the amide I band. 
Based on the studies above, the FEL wavelength at 6.0-6.2 $\mu \mathrm{m}$ might be an optimal wavelength to dissociate polyglutamine aggregate. We prepared aggregates consisting of polyglutamine peptide having 69 glutamine repeats [31]. Electron microscopic analysis revealed that the resultant polyglutamine fibrils formed a number of wires of several hundred nanometers in length (Figure 1A). When we applied irradiation at $5.50 \mu \mathrm{m}$, the fibril form was not largely changed (Figure 1B). Remarkably, irradiation at $6.08 \mu \mathrm{m}$ substantially decreased the fibril form (Figure 1C). The marked conformational change of fibrils after the irradiation at $6.08 \mu \mathrm{m}$ was also analyzed by infrared microscope spectroscopy (Figure 1D). A broad peak at $1660 \mathrm{~cm}^{-1}$ and a shoulder at $1620 \mathrm{~cm}^{-1}$ in amide I region were found in the infrared spectrum of polyglutamine fibrils before the irradiation (red curve). The former peak corresponds to the amide carbonyl bond of the non-fibril form and side-chain of glutamine residue, and the latter shoulder indicates the fibril form, because the resonant frequency of the amide carbonyl group in $\beta$-sheet structure can be generally observed at $1610-1630 \mathrm{~cm}^{-1}$. Before irradiation, proportions of $\alpha$-helix and $\beta$-sheet were calculated to be almost comparable (around 20\%) and lower than those of $\beta$-turn and other conformation. Interestingly, the FEL irradiation at $6.08 \mu \mathrm{m}$ led to decrease in the latter shoulder peak and increase in the former peak (green curve). Quantitatively, the percentage of $\alpha$-helix increased to the level comparable to that of $\beta$-turn or other conformation, while the $\beta$-sheet content decreased to $10 \%$. On the contrary, the spectral pattern was not essentially changed after the irradiation at $5.50 \mu \mathrm{m}$ (blue curve). These results showed that fibril-specific conformation
( $\beta$-sheet) markedly decreased and the fibril form was converted to the non-fibril state specifically after the irradiation at $6.08 \mu \mathrm{m}$ as shown in other protein aggregates.

We further examined the applicability of the FEL irradiation to aggregates inside the cells [31]. Polyglutamine aggregates in the culture medium spontaneously enter $293 \mathrm{~T}$ cells. The cells having polyglutamine aggregates were irradiated and conformational change of the aggregates was examined. The secondary structure mapping by the lattice measurement revealed that the fibril-specific $\beta$-sheet conformation was markedly converted to $\alpha$-helix-dominant conformation by the FEL tuned to amide I band, while the mapping image after irradiation at $5.50 \mu \mathrm{m}$ revealed that the content of $\alpha$-helix was comparatively lower (10-15\%) than those of $\beta$-sheet (ca. 35\%), $\beta$-turn and other conformation (both around $40 \%$ ). Thus, the FEL turned out useful not only for naked aggregates but for the aggregates inside the cells.

\section{Future perspective}

Near-infrared treatment has yielded beneficial outcomes in clinical trials using patients with macular degeneration. The improved vision in the patients was kept for 3-36 months after treatment with no adverse effects [32]. As for polyglutamine diseases, we demonstrated a potency of the FEL at mid-infrared region to dissociate polyglutamine aggregate in a cell line other than neuronal cells [31]. Since polyglutamine aggregates are physiologically located in the neurons
A

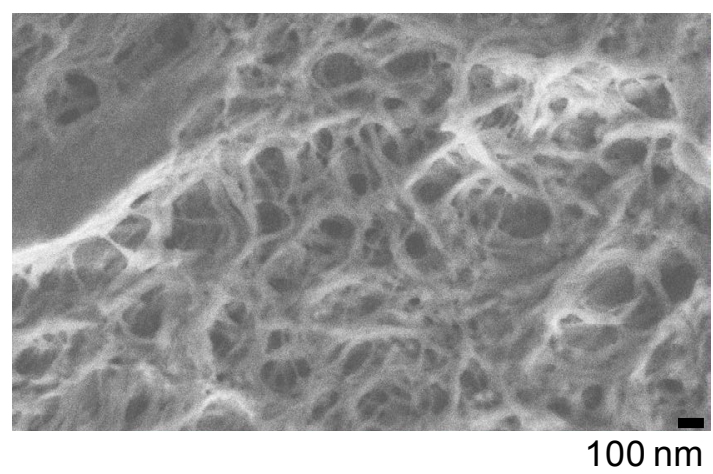

B

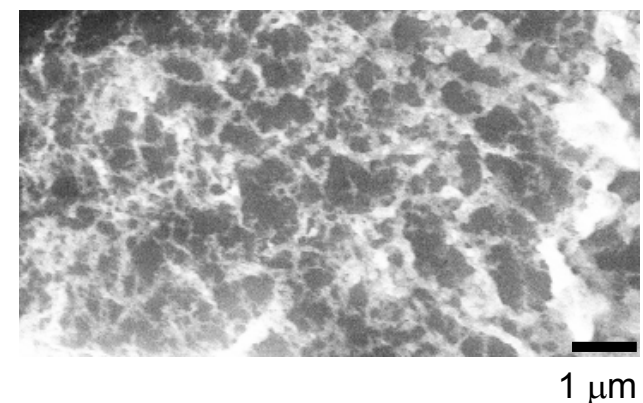

C
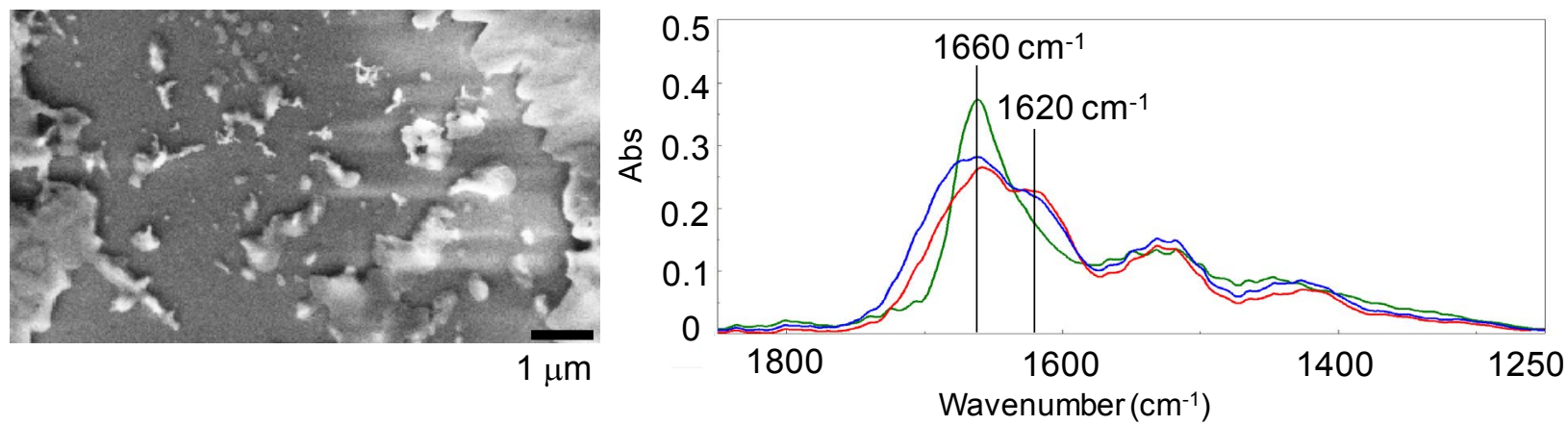

Figure 1.Dissociation of polyglutamine aggregate by FEL irradiation at around $6 \mu \mathrm{m}$. Aggregation of polyglutamine peptide (69 glutamine repeats) in PBS (100 $\mu \mathrm{g} / \mathrm{ml})$ was induced by incubation at $37^{\circ} \mathrm{C}$ for $48 \mathrm{hrs}$. The resultant aggregate was subjected to FEL irradiation and the following analyses.A-C: Scanning-electron microscope analysis. A: Fibrils before the irradiation; B: The fibrils after the irradiation at $5.50 \mu \mathrm{m} ; \mathrm{C}$ : The fibrils after the irradiation at $6.08 \mu \mathrm{m}$. D: Infrared microscope analysis. The infrared absorption spectra were obtained in the mid-infrared range of $700-4000 \mathrm{~cm}^{-1}$. Smoothing and normalization of the spectra were performed on the amide I band $\left(1600-1700 \mathrm{~cm}^{-1}\right)$ using Spectra Manager software. Contents of main conformations in peptide, $\alpha$-helix, $\beta$-sheet, $\beta$-turn, and others were calculated by de-convolution of amide I band $\left(1600-1700 \mathrm{~cm}^{-1}\right)$. Red curve, fibrils before the irradiation; green curve, the fibrils after the irradiation at $6.08 \mu \mathrm{m}$; blue curve, the fibrils after the irradiation at $5.50 \mu \mathrm{m}$. 
in polyglutamine disease brains, the next step is to test usefulness of the FEL for polyglutamine aggregates in cultured neurons. As for possible side effects of the FEL, our measurement using the thermal camera showed that the increase in temperature of the cultured cells induced by this infrared laser irradiation was no greater than $1 \mathrm{~K}$ [31]. Thus, it is not likely that serious cell damages are incurred by heating during irradiation at least under this FEL condition. Future detailed studies using cultured neuronal cells will test whether the FEL disrupts neuronal morphology and functions to establish the safety of the FEL.

\section{Conflict of interest}

There are no conflicts of interest in the content of this paper.

\section{References}

1. Murakoshi Y, Takahashi T, Mihara H (2013) Modification of a small beta-barrel protein, to give pseudo-amyloid structures, inhibits amyloid beta-peptide aggregation. Chemistry 19: 4525-4531.

2. Takahashi T, Mihara $\mathrm{H}$ (2008) Peptide and protein mimetics inhibiting amyloid betapeptide aggregation. AccChem Res 41: 1309-1318. [Crossref]

3. Takahashi T, Ohta K, Mihara H (2010) Rational design of amyloid beta peptide-binding proteins: pseudo-Abeta beta-sheet surface presented in green fluorescent protein binds tightly and preferentially to structured Abeta. Proteins 78: 336-347. [Crossref]

4. Purushothuman S, Johnstone DM, Nandasena C, EerselJv, Ittner LM, et al. (2015) Near infrared light mitigates cerebellar pathology in transgenic mouse models of dementia. Neurosci Lett 591: 155-159. [Crossref]

5. Chung H, Dai T, Sharma SK, Huang YY, Carroll JD, et al. (2012) The nuts and bolts of low-level laser (light) therapy. Ann Biomed Eng 40: 516-533. [Crossref]

6. Meng C, He Z, Xing D (2013) Low-level laser therapy rescues dendrite atrophy via upregulating BDNF expression: implications for Alzheimer's disease. J Neurosci 33: 13505-13517. [Crossref]

7. Purushothuman S, Johnstone DM, Nandasena C, Mitrofanis J, Stone J (2014) Photobiomodulation with near infrared light mitigates Alzheimer's disease-related pathology in cerebral cortex - evidence from two transgenic mouse models. Alzheimers Res Ther 6: 2. [Crossref]

8. Lu Y, Wang R, Dong Y, Tucker D, Zhao N, et al. (2017) Low-level laser therapy for beta amyloid toxicity in rat hippocampus. Neurobiol Aging 49: 165-182. [Crossref]

9. Farfara D, Tuby H, Trudler D, Doron-Mandel E, Maltz L, et al. (2015) Low-level laser therapy ameliorates disease progression in a mouse model of Alzheimer's disease. JMolNeurosci 55: 430-436. [Crossref]

10. Li M, Yang X, Ren J, Qu K, Qu X (2012) Using graphene oxide high near-infrared absorbance for photothermal treatment of Alzheimer's disease. Adv Mater 24: 17221728. [Crossref]

11. Saltmarche AE, Naeser MA, Ho KF, Hamblin MR, Lim L (2017) Significant Improvement in Cognition in Mild to Moderately Severe Dementia Cases Treated with Transcranial Plus Intranasal Photobiomodulation: Case Series Report. Photomed Laser Surg. [Crossref]

12. O'Shea PG, Freund HP (2001) Free-electron lasers. Status and applications. Science 292: 1853-1858. [Crossref]

13. Kawasaki T, Fujioka J, Imai T, Torigoe K, Tsukiyama, K (2014) Mid-infrared freeelectron laser tuned to the amide I band for converting insoluble amyloid-like protein fibrils into the soluble monomeric form. Lasers Med Sci 29: 1701-1707.

14. Manto MU (2005) The wide spectrum of spinocerebellar ataxias (SCAs). Cerebellum 4: 2-6. [Crossref]
15. Taroni F, DiDonato S (2004) Pathways to motor incoordination: the inherited ataxias Nat Rev Neurosci 5: 641-655. [Crossref]

16. Matilla-Duenas A, Goold R, Giunti P (2008) Clinical, genetic, molecular, and pathophysiological insights into spinocerebellar ataxia type 1. Cerebellum 7: 106-114. [Crossref]

17. Robitaille Y, Schut L, Kish SJ (1995) Structural and immunocytochemical features of olivopontocerebellar atrophy caused by the spinocerebellar ataxia type 1 (SCA-1) mutation define a unique phenotype. Acta Neuropathol 90: 572-581. [Crossref]

18. Olson SD, Pollock K, Kambal A, Cary W, Mitchell GM, et al. (2012) Genetically engineered mesenchymal stem cells as a proposed therapeutic for Huntington's disease. MolNeurobiol 45: 87-98. [Crossref]

19. Bonab MM, Sahraian MA, Aghsaie A, Karvigh SA, Hosseinian SM, et al. (2012) Autologous mesenchymal stem cell therapy in progressive multiple sclerosis: an open label study. Curr Stem Cell Res Ther 7: 407-414. [Crossref]

20. Liu J, Han D, Wang Z, Xue M, Zhu L, et al. (2013) Clinical analysis of the treatment of spinal cord injury with umbilical cord mesenchymal stem cells. Cytotherapy 15 : 185-191. [Crossref]

21. Dongmei H, Jing L, Mei X, Ling Z, Hongmin Y, et al. (2011) Clinical analysis of the treatment of spinocerebellar ataxia and multiple system atrophy-cerebellar type with umbilical cord mesenchymal stromal cells. Cytotherapy 13: 913-917. [Crossref]

22. Jin JL, Liu Z, Lu ZJ, Guan DN, Wang C, et al. (2013) Safety and efficacy of umbilical cord mesenchymal stem cell therapy in hereditary spinocerebellar ataxia CurrNeurovasc Res 10: 11-20. [Crossref]

23. Matsuura S, Shuvaev AN, Iizuka A, Nakamura K, Hirai H (2014) Mesenchymal stem cells ameliorate cerebellar pathology in a mouse model of spinocerebellar ataxia type 1. Cerebellum 13: 323-330. [Crossref]

24. Nakamura K, Mieda T, Suto N, Matsuura S, Hirai H (2015) Mesenchymal stem cells as a potential therapeutic tool for spinocerebellar ataxia. Cerebellum 14: 165-170. [Crossref]

25. Mieda T, Suto N, Iizuka A, Matsuura S, Iizuka H, et al. (2016) Mesenchymal stem cells attenuate peripheral neuronal degeneration in spinocerebellar ataxia type 1 knockin mice. J Neurosci Res 94: 246-252. [Crossref]

26. Suto N, Mieda T, Iizuka A, Nakamura K, Hirai H (2016) Morphological and Functional Attenuation of Degeneration of Peripheral Neurons by Mesenchymal Stem Cell-Conditioned Medium in Spinocerebellar Ataxia Type 1-Knock-in Mice. CNS Neuroscience \& Therapeutics 22: 670-676. [Crossref]

27. Kawasaki T, Fujioka J, Imai T, Tsukiyama K (2012) Effect of mid-infrared freeelectron laser irradiation on refolding of amyloid-like fibrils of lysozyme into native form. Protein J 31: 710-716. [Crossref]

28. Kawasaki T, Imai T, Tsukiyama K (2014) Use of a Mid-Infrared Free-Electron Laser (MIR-FEL) for Dissociation of the Amyloid Fibril Aggregates of a Peptide. J Anal Sci Meth Instrum 4: 9-18.

29. Kawasaki T, Yaji T, Imai T, Ohta T, Tsukiyama K (2014) Synchrotron-Infrared Microscopy Analysis of Amyloid Fibrils Irradiated by Mid-Infrared Free-Electron Laser. Am J Anal Chem 5: 384-394.

30. Kawasaki T, Yaji T, Ohta T, Tsukiyama K (2016) Application of mid-infrared freeelectron laser tuned to amide bands for dissociation of aggregate structure of protein. $J$ Synchrotron Radiat 23: 152-157. [Crossref]

31. Kawasaki T, Ohori G, Chiba T, Tsukiyama K, Nakamura K (2016) Picosecond pulsed infrared laser tuned to amide I band dissociates polyglutamine fibrils in cells. Lasers Med Sci 31: 1425-1431. [Crossref]

32. Ivandic BT, Ivandic T (2008) Low-level laser therapy improves vision in patients with age-related macular degeneration. Photomed Laser Surg 26: 241-245. [Crossref]

Copyright: (2017 Nakamura K. This is an open-access article distributed under the terms of the Creative Commons Attribution License, which permits unrestricted use, distribution, and reproduction in any medium, provided the original author and source are credited. 